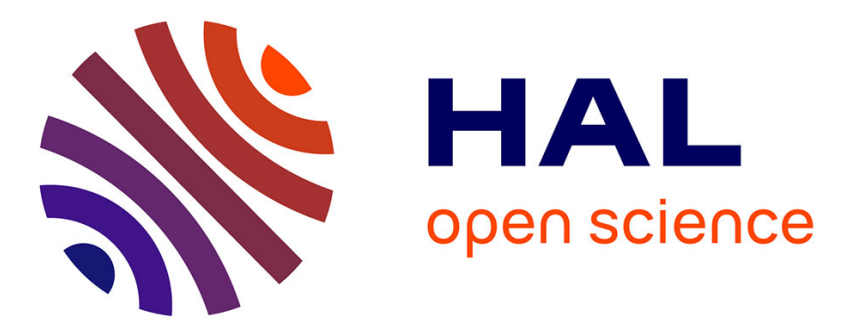

\title{
Optimizing microstructure of a poroelastic layer with cylindrical pores for absorption properties
}

Nikolai Gorbushin, Salah Naili, Vu-Hieu Nguyen

\section{To cite this version:}

Nikolai Gorbushin, Salah Naili, Vu-Hieu Nguyen. Optimizing microstructure of a poroelastic layer with cylindrical pores for absorption properties. Mechanics Research Communications, 2019, 102, pp.103422. 10.1016/j.mechrescom.2019.103422 . hal-03232878

\section{HAL Id: hal-03232878 \\ https://hal.science/hal-03232878}

Submitted on 21 Dec 2021

HAL is a multi-disciplinary open access archive for the deposit and dissemination of scientific research documents, whether they are published or not. The documents may come from teaching and research institutions in France or abroad, or from public or private research centers.
L'archive ouverte pluridisciplinaire HAL, est destinée au dépôt et à la diffusion de documents scientifiques de niveau recherche, publiés ou non, émanant des établissements d'enseignement et de recherche français ou étrangers, des laboratoires publics ou privés.

\section{(ㄷ)(1) $\$$}

Distributed under a Creative Commons Attribution - NonCommercial| 4.0 International 


\title{
Optimizing microstructure of a poroelastic layer with cylindrical pores for absorption properties
}

\author{
Nikolai Gorbushin, Salah Naili, Vu-Hieu Nguyen* \\ Université Paris-Est, Laboratoire Modélisation et Simulation Multi Echelle, MSME UMR 8208 CNRS, \\ 61 avenue du Général de Gaulle, Créteil Cedex 94010, France
}

\begin{abstract}
The present work addresses the problem of multi-scale characterization and optimization of absorption properties of poroelastic materials. Considering the microstructural parameters, we compute the effective properties and field equations within the framework of Biot's theory. Asymptotic homogenization method is used for computing the effective properties which are then applied to solve an acoustic problem. The solution of the latter problem enables the choice of the objective function for the optimization procedure of the optimal design of an unit cell. Preliminary numerical studies show that different skeleton rigidity (soft, stiff or perfectly rigid) may lead to distinct acoustic behavior and optimal parameters for the poroelastic panel.
\end{abstract}

Keywords: Poroelasticity, homogenization, effective properties, acoustic absorption, optimization

\section{Introduction}

Production of new materials requires prior knowledge of the content of components, their mechanical characteristics, geometrical parameters of compounds, and their arrangement inside a solid. Among the huge class of various materials, we focus on poroelastic materials which are widely used as acoustic absorbers and thermal insulators due to their exclusive properties $[1,2]$.

Dynamic behavior of porous materials are usually modeled by Biot's theory [3, 4], which describes the coupling between the solid skeleton and the fluid phase. The Biot model is defined by using of a set of physical parameters such as the elasticity tenor, the permeability tensor, the poroelastic coefficients, etc. Current micromechanical techniques are able to estimate all mentioned properties before the production stage. Among the most common techniques are the average-field theory [5] and the asymptotic homogenization $[6,7,8]$. A comparison between these two techniques was reported in [9]. The homogenization theory, which is more rigorous from the mathematical viewpoint, will be employed in the present study.

It is observed that the temperature fluctuation may significantly influence the acoustic performance of porous materials [1]. Thermal effects on the macroscopic behavior of a porous material can be modeled by means of the homogenization procedure as well $[10,11]$. When the variation of temperature in the solid skeleton is negligible in comparison with that in the fluid phase, the derivation of

\footnotetext{
* Corresponding author

Email address: vu-hieu.nguyen@u-pec.fr (Vu-Hieu Nguyen)
}

macroscopic equations could be simplified. This assumption is used in [12] within the asymptotic homogenization procedure which are developed for improvement of existing acoustic absorbers. Acoustic absorption in materials with double porosity and rigid skeleton is analyzed with accounting to temperature effects in [13].

In the context of design of poroelastic isolation panels, optimal macroscopic acoustic absorption and/or transmission loss depend not only on the characteristics of bulk porous materials, but also on the structural parameters (e.g the panel's thickness). Moreover, composite panels made of a mixture of different poroelastic materials may also improve acoustic performances in absorption or transmission losses [14]. This work aims at developping a homogenization-based procedure to design a poroelastic panel with an optimal acoustic absorption by modifying microstructural cell parameters. To the best of the authors' knowledge, in the literature, still few works have been conducted on optimizing the microsctructure of porous materials in the context of studying global acoustic absorption of plates [15, 16]. Basically, by varying topological parameters of an unit cell, the optimal macroscopic acoustic absorption could be achieved. For example, various arrangements of cylindrical inclusions [17, 18] or resonant inclusions with a split ring shape cross section which perform as Helmholtz resonator [19, 20] can significantly increase the acoustic properties of a bulk material. However, the solid skeleton are assumed to be perfectly rigid for most mentioned studies whereas absorption properties may significantly depend on skeleton stiffness.

This study serves as a preliminary analysis by considering a simple geometry of unit cell. After the description of 
the problem in Section 2, Section 3 presents a homogenization procedure for estimating effective poroelastic properties by taking into account thermal effects. Next, Section 4 presents the acoustic problem at the macroscopic scale and semi-analytical formulation for determining the absorption coefficient. In Section 5, we study the dependence of the absorption coefficient on the cell size and on the porosity, which appear to be the design parameters for the optimization routine. Two kinds of solid skeleton materials, which have very different elastic properties form each to other, will be studied.

\section{Description of the problem}

Let us consider a poroelastic layer of thickness $d$ which is described in the Cartesian coordinate system with an orthogonal basis $\left(\mathbf{e}_{1}, \mathbf{e}_{2}, \mathbf{e}_{3}\right)$ and the position vector $\mathbf{x}=$ $\left(x_{1}, x_{2}, x_{3}\right)$ (Fig. 1). The layer has a rigid backing at one side and surrounded by the air from another. In the present study, we only consider the normal incident wave in the $\mathbf{e}_{3}$-direction, which corresponds to the testing of materials in impedance tubes [1]. The incident plane pressure wave, denoted by $p_{i} \exp \left(i \omega t-i q_{0} x_{3}\right)$, with the wavenumber $q_{0}=c_{0} / \omega$, angular frequency $\omega$ and $i^{2}=-1$, hits the porous layer and reflects from it. The reflected wave field is defined as $p_{r} \exp \left(i \omega t+i q_{0} x_{3}\right)$.

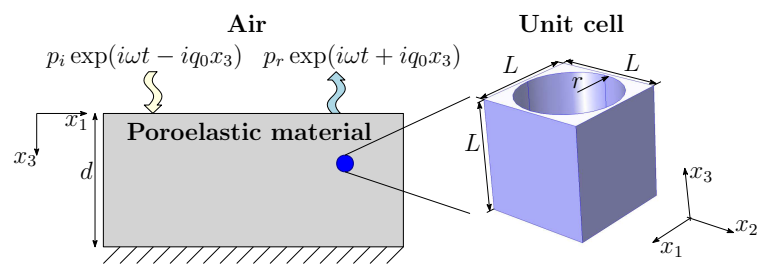

Figure 1: Acoustic problem for a porous layer of thickness $d$ with microstructure formed of an unit cell with cylindrical inclusion

The macroscopic material properties of the porous material are not yet known and have to be found from a homogenization procedure. We assume that the poroelastic material has periodic microstructure. In this work, we consider a unit cell of size $L \times L \times L$ with the cylindrical pore filled with air whose radius is $r$ and height is $L$ along the $\mathbf{e}_{3}$-direction (Fig. 1). Only harmonic problem will be considered.

In what follows, symbols "." and ":" refer to the scalar product and the double contraction, respectively. Symbols $\nabla$ and $\nabla \cdot$ stands for the gradient and divergence operators, respectively. $\nabla^{s}$ denoted the symmetric part of the gradient operator. The dot notation above a vector/scalar field represents a time derivative.

\section{Homogenisation procedure}

The equations of the multiphysical problem under consideration at microscale are stated separately for solid and fluid phases. Considering a porous material with periodic microstructure, material properties in such material vary periodically with a period of the order of a small scale parameter $\varepsilon=l_{m i c} / l_{m a c}$, where $l_{m i c}$ and $l_{m a c}$ are characteristic lengths at micro- and macroscales. We suppose that the material fills the tridimensional domain $\Omega$ with the boundary $\partial \Omega$. The domain $\Omega$ is generated by a periodic translation of a cubic unit cell $Y=\prod_{i=1}^{3}[0, L]$ and is composed of the volume occupied by solid $\Omega_{s}^{\varepsilon}$ and fluid $\Omega_{f}^{\varepsilon}$ phases separated by the interface $\Gamma_{f s}^{\varepsilon}$ such as $\Omega=\Omega_{s}^{\varepsilon} \cup \Omega_{f}^{\varepsilon} \cup \Gamma_{f s}^{\varepsilon}$. Superscript ${ }^{\varepsilon}$ signifies the scale-dependence. Similarly, the unit cell is composed of the solid $Y_{s}$ and fluid $Y_{f}$ phases with their common boundary $\Gamma_{f s}: Y=Y_{s} \cup Y_{f} \cup \Gamma_{f s}$. The boundaries $\partial \Omega_{s}^{\varepsilon}$ and $\partial \Omega_{f}^{\varepsilon}$ have outward unit normal vectors $\mathbf{n}^{s}$ and $\mathbf{n}^{f}$, respectively, with $\mathbf{n}^{s}=-\mathbf{n}^{f}$ on $\Gamma_{f s}$.

The state of poroelastic material is described by the displacement field inside the solid, $\mathbf{u}^{s, \varepsilon}$, velocity of the fluid, $\dot{\mathbf{u}}^{f, \varepsilon}$, deviations of pressure, density and temperature of the fluid $p^{\varepsilon}, \delta^{f, \varepsilon}$ and $\tau^{\varepsilon}$, respectively, from their equilibrium values. Stresses inside each of the phases, solid and fluid, are denoted by $\boldsymbol{\sigma}^{s, \varepsilon}$ and $\boldsymbol{\sigma}^{f, \varepsilon}$, respectively. The solid phase $\Omega_{s}^{\varepsilon}$ is characterized by the elasticity tensor $\mathbb{C}$ and density $\rho^{s, \varepsilon}$. By neglecting the body force, the governing equations of the linear elastic solid are given by:

$$
\begin{gathered}
\nabla \cdot \boldsymbol{\sigma}^{s, \varepsilon}=-\rho^{s} \omega^{2} \mathbf{u}^{s, \varepsilon}, \quad \text { in } \Omega_{s}^{\varepsilon}, \\
\boldsymbol{\sigma}^{s, \varepsilon}=\mathbb{C} \nabla^{s} \mathbf{u}^{s, \varepsilon} .
\end{gathered}
$$

Let $\rho^{f}, T_{0}$ and $P_{0}$ be values of density, temperature and pressure in the fluid phase, respectively, at the equilibrium state. The fluid phase is described by the linearized Navier-Stokes and ideal gas state equations:

$$
\begin{gathered}
i \omega \delta^{f, \varepsilon}+\rho^{f} \nabla \cdot \dot{\mathbf{u}}^{f, \varepsilon}=0, \quad \text { in } \Omega_{f}^{\varepsilon}, \\
\frac{p^{\varepsilon}}{P_{0}}=\frac{\delta^{f, \varepsilon}}{\rho^{f}}+\frac{\tau^{\varepsilon}}{T_{0}}, \quad \text { in } \Omega_{f}^{\varepsilon}, \\
i \omega \rho_{0} \dot{\mathbf{u}}^{f, \varepsilon}=\nabla \cdot \boldsymbol{\sigma}^{f, \varepsilon}, \quad \text { in } \Omega_{f}^{\varepsilon}, \\
\boldsymbol{\sigma}^{f, \varepsilon}=-p^{\varepsilon} \mathbf{I}+2 \mu \nabla^{s} \dot{\mathbf{u}}^{f, \varepsilon}-\frac{2}{3} \mu\left(\nabla \cdot \dot{\mathbf{u}}^{f, \varepsilon}\right) \mathbf{I},
\end{gathered}
$$

where $\mu$ is the dynamic viscosity of the fluid and $\mathbf{I}$ is the unit tensor. The second equation in (2) is the state equation for an ideal gas, which turns out to be a good approximation for the characterization of air. This state equation is widely adopted for the similar problems [1, 21, 12] and derived with the assumption $\kappa \ll \rho^{f} \omega C_{v}^{f} l_{\text {mac }}^{2}$, where $\kappa$ and $C_{v}^{f}$ are respectively the isotropic thermal conductivity and the heat capacity of fluid under constant volume [22], which holds for the present case as well.

Equations (1) and (2) are complemented by the heat equation for the fluid phase. Let $\mathbf{q}^{\varepsilon}$ be the heat flux, then:

$$
\begin{gathered}
-\nabla \cdot \mathbf{q}^{\varepsilon}=i \omega \rho^{f} C_{v}^{f} \tau^{f, \varepsilon}+\left(i \omega \rho_{0} R_{s p} \tau^{\varepsilon}-i \omega p^{\varepsilon}\right), \text { in } \Omega_{f}^{\varepsilon}, \\
\mathbf{q}^{\varepsilon}=-\boldsymbol{\kappa}^{\varepsilon} \nabla \tau^{\varepsilon},
\end{gathered}
$$

where $R_{s p}$ is the specific gas constant and $\boldsymbol{\kappa}$ is the thermal conductivity second-order tensor. For an ideal gas, 
Mayer's relation reads: $C_{p}^{f}-C_{v}^{f}=R_{s p}$, where $C_{p}^{f}$ is the heat capacity under constant pressure. One may also recall that $C_{p}^{f}-C_{v}^{f}=P_{0} /\left(\rho^{f} T_{0}\right)$ is true for an ideal gas.

Finally, the system of equations is closed with the boundary conditions:

$$
\begin{gathered}
i \omega \mathbf{u}^{s, \varepsilon}=\dot{\mathbf{u}}^{f, \varepsilon}, \quad \text { on } \Gamma_{f s}^{\varepsilon}, \\
\boldsymbol{\sigma}^{s, \varepsilon} \mathbf{n}^{s}=\boldsymbol{\sigma}^{f, \varepsilon} \mathbf{n}^{s}, \quad \text { on } \Gamma_{f s}^{\varepsilon}, \\
\tau^{\varepsilon}=0, \quad \text { on } \Gamma_{f s}^{\varepsilon} .
\end{gathered}
$$

In what follows, the vector $\mathbf{x}=\left(x_{1}, x_{2}, x_{3}\right)$ refers to the macroscopic coordinates whereas the microscopic coordinates are associated with the macroscopic one through the relation $\mathbf{y}=\varepsilon^{-1} \mathbf{x}$. These two scales are assumed to be well separated so that homogenization technique with double-scale asymptotic expansion can be performed. The differential operators $\nabla_{x}$ and $\nabla_{y}$ correspond to differentiation with respect to $\mathbf{x}$ and $\mathbf{y}$, respectively. Porosity of material is equal to $\phi=\left|Y_{f}\right| /|Y|$ and the averaging operators are defined as $\langle()\rangle=.1 /|Y| \int_{Y}() d$.$V , where |Y|$ denotes the volume of $Y$. We also introduce the functional space $\mathcal{C}_{\#}\left(Y_{s, f}\right)$ of sufficiently differential functions on $Y_{s, f}$ and $Y$-periodic. Moreover, the infinitesimal strain tensor is defined by: $\boldsymbol{\epsilon}(\mathbf{u})=\nabla^{s} \mathbf{u}$.

The major mechanisms that provide high acoustic absorption in porous materials are viscous damping and thermal dissipation which happens close to the solid-fluid interface. The viscous depth $\delta_{v}=\left(2 \mu /\left(\rho^{f} \omega\right)\right)^{1 / 2}$ and thermal skin depth $\delta_{t}=\left(\kappa /\left(\rho^{f} \omega C_{p}^{f}\right)\right)^{1 / 2}$ define characteristic length scales which attribute these effects. In the present study, we consider the pores saturated with air and the frequency range is chosen such as $O\left(\delta_{v, t}^{2} / l_{\text {mac }}^{2}\right)=O\left(\varepsilon^{2}\right)$, where $O(\star)$ is big-oh notation, which is used to describe the asymptotic behavior of the function. Basing these remarks, the scaled constitutive equations for the fluid phase may be expressed by:

$$
\begin{gathered}
\boldsymbol{\sigma}^{f}=-p \mathbf{I}+2 \varepsilon^{2} \mu \nabla_{x}^{s} \dot{\mathbf{u}}^{f}-\frac{2}{3} \varepsilon^{2} \mu\left(\nabla_{x} \cdot \dot{\mathbf{u}}^{f}\right) \mathbf{I}, \\
\mathbf{q}=-\varepsilon^{2} \boldsymbol{\kappa} \nabla_{x} \tau .
\end{gathered}
$$

The derivation of cell problems and macroscopic state equations can be obtained when $\mathbf{u}^{s}, \dot{\mathbf{u}}^{f}, p, \tau$ and $\delta^{f}$ are expanded in power series of $\varepsilon$ and then substituted into corresponding governing equations. The reader can follow derivations presented in [12] to obtain the results. For the unit cell of interest, the analytical results for the effective permeability and compressibility could be found [21, 23] and only effective properties corresponding to Biot's model should be computed. The remaining properties are found from solving two cell problems through the finite element method (FEM).

The first cell problem in its weak form reads: Find functions $\chi^{k l} \in \mathcal{C}_{\#}\left(Y_{s}\right)$ such as:

$$
\begin{gathered}
\int_{Y_{s}}\left[C_{i j p q} \epsilon_{p q}\left(\chi^{k l}\right)+C_{i j k l}\right] \epsilon_{i j}\left(\delta \chi^{k l}\right) d V=0, \\
\forall \delta \chi^{k l} \in \mathcal{C}_{\#}\left(Y_{s}\right) .
\end{gathered}
$$

in which the Einstein summation convention is used. Equation (6) refers to the Neumann boundary value problem and its solution is determined up to an arbitrary constant. For this reason, the zero average condition is additionally stated: $\left\langle\chi^{k l}\right\rangle=\mathbf{0}$.

The formulation of the second cell problem to solve can be presented as: Find $\boldsymbol{\eta} \in \mathcal{C}_{\#}\left(Y_{s}\right)$ such as:

$$
\int_{Y_{s}} C_{i j k l} \epsilon_{k l}(\boldsymbol{\eta}) \epsilon_{i j}(\delta \boldsymbol{\eta}) d V=\int_{Y_{f s}} \delta \boldsymbol{\eta} \cdot \mathbf{n}^{s} d S, \quad \forall \delta \boldsymbol{\eta} \in \mathcal{C}_{\#}\left(Y_{s}\right) .
$$

For the same reason as in the previous case, vector function $\boldsymbol{\eta}$ is subjected to the zero average constraint: $\langle\boldsymbol{\eta}\rangle=\mathbf{0}$.

The homogenization method provides constitutive relations for averaged fields. These equations are written within the framework of Biot's theory of poroelasticity. First, we introduce the total stress tensor:

$$
\boldsymbol{\sigma}=\left\{\begin{array}{l}
-p \mathbf{I}, \quad \text { in } \Omega_{f} \\
\boldsymbol{\sigma}^{s}, \quad \text { in } \Omega_{s}
\end{array}\right.
$$

The constitutive equations yield:

$$
\begin{gathered}
\boldsymbol{\sigma}=\mathbb{C}^{*}: \boldsymbol{\epsilon}-\hat{\mathbf{B}} p, \\
\nabla \cdot \phi\left(\dot{\mathbf{u}}^{f}-i \omega \mathbf{u}^{s}\right)=-i \omega \hat{\mathbf{B}}: \boldsymbol{\epsilon}-i \omega \hat{M} p,
\end{gathered}
$$

where $\mathbb{C}^{*}$ is the drained effective stiffness fourth-order tensor, $\hat{\mathbf{B}}$ is the second-order Biot coefficient tensor, representing the fluid-solid coupling, and $\hat{M}$ is a scalar coefficient, characterizing modified fluid compressibility due to the solid skeleton. In some studies, $1 / \hat{M}$ is referred to as the Biot modulus and $\hat{M}$ as a storage coefficient. These quantities are defined by means of the solutions of cell problems as follows:

$$
\begin{gathered}
C_{i j k l}^{*}=\left\langle C_{i j p q}\left[\epsilon_{p q}\left(\chi^{k l}\right)+\delta_{p k} \delta_{q l}\right]\right\rangle, \\
\hat{B}_{i j}=\phi \delta_{i j}+B_{i j}, B_{i j}=-\left\langle\epsilon_{k k}\left(\chi^{i j}\right)\right\rangle=\left\langle C_{i j k l} \epsilon_{k l}(\boldsymbol{\eta})\right\rangle, \\
\hat{M}=\phi \alpha+M, M=\left\langle\epsilon_{k k}(\boldsymbol{\eta})\right\rangle,
\end{gathered}
$$

where $\alpha=\alpha(\omega)$ is the effective fluid compressibility and is a frequency-dependent function due to the thermal fluctuations. The macroscopic field equations become:

$$
\begin{aligned}
& -\bar{\rho} \omega^{2} \mathbf{u}^{s}-\rho^{f} \omega^{2} \mathbf{w}^{f}=\nabla_{x} \cdot \boldsymbol{\sigma}, \\
& i \omega \mathbf{w}^{f}=-\mathbf{K}\left(\nabla_{x} p-\rho^{f} \omega^{2} \mathbf{u}^{s}\right) .
\end{aligned}
$$

In the last equations, the following quantities are defined $\bar{\rho}=(1-\phi) \rho^{s}+\phi \rho^{f}$ and $\mathbf{w}^{f}=\phi\left(\mathbf{u}^{f}-\mathbf{u}^{s}\right)$. The frequency dependent $\mathbf{K}=\mathbf{K}(\omega)$ represents the effective permeability second-order tensor. One can easily conclude from the geometry of the cell shown in Fig. 1 that there is only one non-zero element of permeability tensor: $K_{i j}=k \delta_{i 3} \delta_{j 3}$, in which $k$ may be analytically calculated by [21, 23]:

$$
\begin{gathered}
k=-\frac{\phi r^{2}}{\mu} \frac{1}{\eta^{2}} F(\eta), \quad \eta=i r \sqrt{\frac{i \omega \rho^{f}}{\mu}}, \\
F(\eta)=-\frac{J_{2}(\eta)}{J_{0}(\eta)}=1-\frac{2}{\eta} \frac{J_{1}(\eta)}{J_{0}(\eta)},
\end{gathered}
$$


where $J_{m}$ designates the Bessel function of the first kind of order $m$, for $m=0,1$ and 2.The analytical expression for the effective compressibility is [21]:

$$
\alpha=\frac{\gamma-(\gamma-1) F\left(\eta^{\prime}\right)}{\gamma P_{0}}, \quad \eta^{\prime}=i r \sqrt{\frac{i \omega \rho^{f} C_{p}^{f}}{\kappa}},
$$

where the function $F\left(\eta^{\prime}\right)$ is taken from the previous expression and $\gamma=C_{p}^{f} / C_{v}^{f}$ is the ratio of constant pressure and volume heat capacities.

\section{Absorption coefficient}

Referring to the setting shown in Fig. 1, the system (11) may be solved by applying the following boundary conditions:

$$
\begin{gathered}
\sigma_{33}=-p^{(n)}, p=p^{(n)}, u_{3}^{s}+w_{3}^{f}=\frac{1}{\rho^{f} \omega^{2}} \frac{\partial p^{(n)}}{\partial x_{3}} \quad \text { for } x_{3}=0, \\
u_{3}^{s}=w_{3}^{f}=0 \quad \text { for } x_{3}=d,
\end{gathered}
$$

where $p^{(n)}=p_{i} \exp \left(i \omega t-i q_{0} x_{3}\right)+p_{r} \exp \left(i \omega t+i q_{0} x_{3}\right)$ is the pressure field of the air outside the layer. In the further analysis, we drop the factor $\exp (i \omega t)$ which appears in every function below. In the case of the normal incident angle, it is sufficient to consider only $u_{3}^{s}$ and $w_{3}^{f}$. The constitutive laws reduce to:

$$
\left(\begin{array}{c}
\sigma_{33} \\
-p
\end{array}\right)=\mathbb{T}\left(\begin{array}{c}
\partial u_{3}^{s} / \partial x_{3} \\
\partial w_{3}^{f} / \partial x_{3}
\end{array}\right), \quad \mathbb{T}=\left(\begin{array}{cc}
C_{33}^{U} & C^{b} \\
C^{b} & C^{M}
\end{array}\right),
$$

where $C_{33}^{U}=C_{33}^{*}+\frac{1}{\hat{M}} \hat{B}_{33}^{2}, C^{b}=\frac{1}{\hat{M}} \hat{B}_{33}, C^{M}=\frac{1}{\hat{M}}$. Seeking the solution in the form $\left(u_{3}^{s}, w_{3}^{f}\right)^{T}=\mathbf{V} \exp \left(i \lambda x_{3}\right)$ and plugging it into (11) leads to the eigenvalue problem:

$$
\left[\mathbb{T} \lambda^{2}-\omega^{2}\left(\begin{array}{cc}
\bar{\rho} & \rho^{f} \\
\rho^{f} & \tilde{\rho}_{3}
\end{array}\right)\right] \mathbf{V}=0
$$

where $\tilde{\rho}_{3}(\omega)=-i /\left(\omega K_{33}(\omega)\right)$ is the component of the dynamic density in the $x_{3}$-direction, $\lambda$ and $\mathbf{V}$ are the eigenvalue and eigenvector, respectively. Solving the characteristic equation of the problem (16) gives:

$$
\lambda_{1,2}=\omega \sqrt{\frac{a_{1} \pm \sqrt{a_{1}^{2}-4 a_{0} a_{2}}}{2 a_{2}}}
$$

where $a_{2}=C_{33}^{U} C^{M}-\left(C^{b}\right)^{2}, a_{1}=C_{33}^{U} \tilde{\rho}_{3}+C^{M} \bar{\rho}-2 C^{b} \rho^{f}$, $a_{0}=\left(\tilde{\rho}_{3} \bar{\rho}-\left(\rho^{f}\right)^{2}\right)$.

The solution that corresponds to propagating waves in the $x_{3}$-direction is related to values $-\lambda_{1,2}$ whereas the waves propagating in the opposite direction are characterized by roots $\lambda_{1,2}$. Eigenvectors can be chosen to be:

$$
\mathbf{V}_{1,2}=\left(\begin{array}{c}
1 \\
\mu_{1,2}
\end{array}\right) \quad \text { where } \mu_{1,2}=-\frac{C^{b} \lambda_{1,2}^{2}-\omega^{2} \rho^{f}}{C^{M} \lambda_{1,2}^{2}-\omega^{2} \tilde{\rho}_{3}}
$$

The solution of the problem can be expressed as:

$$
\left(\begin{array}{c}
u_{3}^{s} \\
w_{3}^{f} \\
\sigma_{33} \\
-p
\end{array}\right)=\left[\begin{array}{cc}
\mathbf{S}_{1} & \mathbf{S}_{1} \\
-\mathbf{S}_{2} & \mathbf{S}_{2}
\end{array}\right]\left[\begin{array}{cc}
\mathbf{D}^{+}\left(x_{3}\right) & \mathbf{0} \\
\mathbf{0} & \mathbf{D}^{-}\left(x_{3}\right)
\end{array}\right]\left(\begin{array}{l}
\mathbf{A}^{+} \\
\mathbf{A}^{-}
\end{array}\right)
$$

where $\mathbf{S}_{1}=\left[\mathbf{V}_{1}, \mathbf{V}_{2}\right], \mathbf{S}_{2}=\left[i \lambda_{1} \mathbb{T} \mathbf{V}_{1}, i \lambda_{2} \mathbb{T} \mathbf{V}_{2}\right], \mathbf{D}^{ \pm}\left(x_{3}\right)=$ $\operatorname{diag}\left(\exp \left(\mp i \lambda_{1} x_{3}\right), \exp \left(\mp i \lambda_{2} x_{3}\right)\right)$, with in the $\operatorname{diag}()$ operator only the diagonal elements are given. Constant vectors $\mathbf{A}^{ \pm}=\left(A_{1}^{ \pm}, A_{2}^{ \pm}\right)^{T}$ are to be determined from the boundary conditions. The condition of rigid backing at $x_{3}=d$ yields: $A_{1,2}^{-}=-A_{1,2}^{+} \exp \left(-2 i \lambda_{1,2} d\right)$. Let $\dot{u}(0)$ be the velocity of the external air at $x_{3}=0$ and $u_{3}^{s}(0)+w_{3}^{f}(0)=\dot{u}(0) /(i \omega)$. The definition of the surface impedance is $Z=p^{(n)}(0) / \dot{u}(0)$. The application of remaining boundary conditions provides the system of linear equations:

$$
\left(\begin{array}{ccc}
\left(1+\mu_{1}\right)\left(1-e_{1}^{-}\right) & \left(1+\mu_{2}\right)\left(1-e_{2}^{-}\right) & -1 \\
z_{11}\left(1+e_{1}^{-}\right) & z_{12}\left(1+e_{2}^{-}\right) & -Z \\
z_{21}\left(1+e_{1}^{-}\right) & z_{22}\left(1+e_{2}^{-}\right) & -Z
\end{array}\right)\left(\begin{array}{c}
A_{1}^{+} \\
A_{2}^{+} \\
\dot{u}(0)
\end{array}\right)=\mathbf{0}
$$

where $z_{1 j}=\left(\lambda_{j} / \omega\right)\left(C_{33}^{U}+C^{b} \mu_{j}\right), z_{2 j}$ $\left(\lambda_{j} / \omega\right)\left(C_{b}+C^{M} \mu_{j}\right), e_{j}^{-}=\exp \left(-2 i \lambda_{j} d\right)$ for $j=1,2$.

The condition for non-trivial solutions of the system (20) to exist imposes that the determinant of the matrix is zero, leading to an explicit expression for the surface impedance:

$$
Z(\omega)=i \frac{z_{12} z_{21}-z_{11} z_{22}}{z_{1}-z_{2}}
$$

where $z_{j}=\frac{\left(z_{22}-z_{12}\right)\left(z_{21}-z_{11}\right)}{z_{2 j}-z_{1 j}}\left(1+\mu_{j}\right) \tan \left(\lambda_{j} d\right)(j=$ $1,2)$. In the case of the rigid skeleton, there is an analytical expression for the surface impedance $[1,24]$ :

$$
Z^{(r)}(\omega)=-i Z_{c}(\omega) \cot \left(k_{c}(\omega) d\right)
$$

where $Z_{c}(\omega)=\sqrt{\frac{\tilde{\rho}_{3}(\omega)}{\phi \alpha(\omega)}}, k_{c}(\omega)=\omega \sqrt{\phi \tilde{\rho}_{3}(\omega) \alpha(\omega)}$.

The absorption coefficient can be then computed by:

$$
\alpha_{a}(\omega)=1-\left|\frac{Z-Z_{0}}{Z+Z_{0}}\right|^{2}, \quad \text { where } Z_{0}=\rho^{f} c_{0} .
$$

\section{Numerical results}

\subsection{Effective elastic properties}

In this study, we consider two kinds of matrix materials with different rigidities. The first one is epoxy which is widely used in the production of composites and can be used for the construction of cellular materials via $3 \mathrm{D}$ printing [25]. The elastic properties are isotropic with Young's modulus $E_{0}=3.5 \mathrm{GPa}$, Poisson's ratio $\nu_{0}=0.33$ and density $\rho^{s}=1540 \mathrm{~kg} / \mathrm{m}^{3}$. Another material of interest is silicone rubber which has a significantly lower 
Young's modulus and has been already used for acoustic applications [26]. The properties of silicone rubber are $E_{0}=1.2 \mathrm{MPa}, \nu_{0}=0.47$ and $\rho^{s}=1300 \mathrm{~kg} / \mathrm{m}^{3}$.

Pores are assumed to be saturated with air with the following properties: $\mu=1.71 \times 10^{-5} \mathrm{~Pa} \cdot \mathrm{s}, \rho^{f}=$ $1.293 \mathrm{~kg} / \mathrm{m}^{3}, c_{0}=331.6 \mathrm{~m} / \mathrm{s}, \kappa=2.41 \times 10^{-2} \mathrm{~W} / \mathrm{m} . \mathrm{K}$, $C_{p}=1006 \mathrm{~J} /(\mathrm{kg} \cdot \mathrm{K}), \gamma=1.403$ at $T_{0}=0^{\circ} \mathrm{C}$ and $P_{0}=10^{5} \mathrm{~Pa}$. The heat conductivity is isotropic in this case and is given by $\kappa=\kappa \mathbf{I}$.

a)

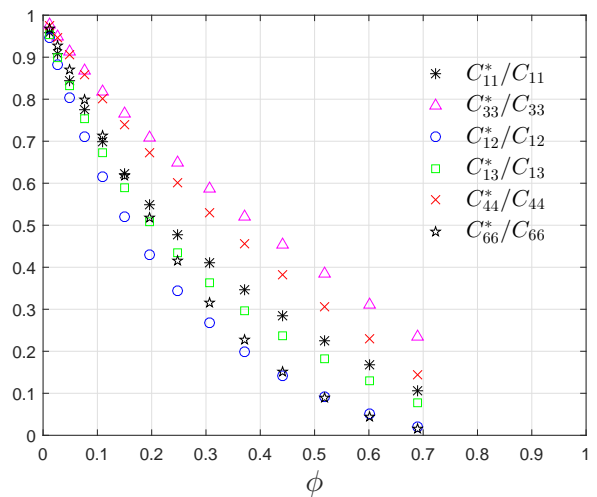

b)

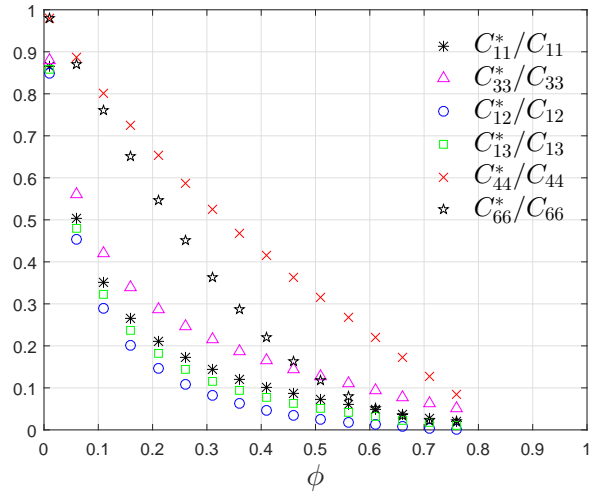

Figure 2: Effective elastic properties in Voigt's notations for configuration of Fig. 1: (a) epoxy matrix material; (b) silicone rubber matrix material.

Effective elastic properties computed by the homogenization approach are plotted in Fig. 2a) for the epoxy matrix and in Fig. 2b) for the silicone rubber matrix. For both materials, we observe the monotonic dependence of the effective elastic constants on porosity. For epoxy matrix, we observe that ratio $C_{33}^{*} / C_{33}$ remains higher than $C_{44}^{*} / C_{44}$ and $C_{66}^{*} / C_{66}$ whereas for the more compliant silicone rubber matrix this pattern is reversed. Next section will estimate of acoustic performances of these homogenized materials and will show the consequences of effective poroelastic properties of the media.

\subsection{Optimal cell size and porosity for absorption}

We are interested in optimal parameters that enable us to design a porous material with the best absorption properties for fixed values of matrix material properties. For the optimization procedure, we focus on the low frequency band. Particularly, by considering a $1 / 3$ octave that includes a set $\mathcal{S}$ of 12 frequencies (in $\mathrm{kHz}$ ) [27]:

$$
\mathcal{S}=\{0.2,0.25,0.315,0.4,0.5,0.63,0.8,1,1.25,1.6,2,2.5\},
$$

we define a parameter $J$ which is the average of absorption coefficient at these frequencies, see e.g. [27]:

$$
J=\frac{1}{12} \sum_{j=1}^{12} \alpha_{a}\left(\omega_{j}\right)
$$

where the $\omega_{j}$, for $j=1, \ldots, 12$, are the values of the set $\mathcal{S}$. The functional $J$ is defined as the objective function and we try to find its maximum over the range of design parameters. We take $\phi$ and $L$ as design parameters for the present study and solve the optimization problem for fixed values of $d$. Effective poroelastic properties involved in Biot's model are not affected by the change of $L$ but remain dependent on the porosity. On the other hand, the effective permeability and compressibility can drastically vary for different values of $L$. The problem is stated as follows:

$$
\min _{\phi, L}-J \text { such that }\left\{\begin{array}{l}
0.01 \leq \phi \leq 0.78 \\
10 \mu \mathrm{m} \leq L \leq 1000 \mu \mathrm{m} .
\end{array}\right.
$$

It is worth noting that there is a maximum value for the porosity $\phi=\pi / 4 \sim 0.785$, when the cylindrical pore touches the walls of the solid matrix. Thus, the upper bound for $\phi$ is chosen according to this limiting value. The upper bound for $L$ is chosen in accordance with the homogenization procedure when the separation of scales is required.

The solution of the constrained optimisation problem is obtained by a direct algorithm so-called the pattern search algorithm [28] which does not require the calculations of gradient. In this study, we used the function patternsearch in Matlab which showed that the convergence could be obtained reasonably fast. The initial point for this method is given by the middle of the grid $(\phi, L)$. The optimisation is performed through iterations when the sequence of nonincreasing function values is obtained [29]. The method is characterised by the mesh of step size $\Delta$ which is estimated in accordance with the constraints introduced in Eq. (25).

The results of optimization procedure are presented in Tab. 1. One can notice that for each value of $d$, the optimal values of $J$ are unchanged by using rigid or epoxy frame models. The absorption coefficient for rigid and epoxy frames appears to be indistinguishable for the studied range of frequencies and $d=1,2,5,10 \mathrm{~cm}$. The results corresponding to the optimal cell geometry are shown in Fig. 3a). There is a localized jump in the absorption coefficient of a porous layer with the epoxy frame for $d=20$ $\mathrm{cm}$, see Fig. 3b). The presence of similar peaks in surface impedance for the elastic frame is also observed [1, sec.6.6]. It is attributed to the resonance close to a quarter of the wavelength of the frame borne wave, generated by the dynamics of the elastic skeleton. At this wavelength, the air 
velocity at the free boundary of the layer reaches its maximum value manifesting a sudden change of the absorption.

\begin{tabular}{|c|c|c|c|}
\hline \multicolumn{4}{|c|}{ Rigid / Epoxy / Silicone rubber } \\
\hline$d(\mathrm{~cm})$ & $L_{\text {opt }}(\mu \mathrm{m})$ & $\phi_{\text {opt }}$ & $J_{\text {opt }}$ \\
\hline 1 & $13 / 13 / 998$ & $0.78 / 0.78 / 0.03$ & $0.03 / 0.03 / 0.06$ \\
\hline 2 & $28 / 28 / 998$ & $0.78 / 0.78 / 0.3$ & $0.06 / 0.06 / 0.18$ \\
\hline 5 & $80 / 80 / 472$ & $0.78 / 0.78 / 0.24$ & $0.14 / 0.14 / 0.27$ \\
\hline 10 & $204 / 204 / 762$ & $0.78 / 0.78 / 0.19$ & $0.3 / 0.3 / 0.36$ \\
\hline 20 & $255 / 255 / 650$ & $0.78 / 0.78 / 0.31$ & $0.42 / 0.42 / 0.49$ \\
\hline
\end{tabular}

Table 1: Results of optimization procedure for a porous layer as shown in Fig. 1; These results are given for the perfectly rigid, epoxy and silicone rubber frames and separated by "/".

It turns out that optimal porosity is the same for all considered cases and takes an extreme value $(\phi=0.78)$ for the possible interval. On the contrary, the results for optimal $L$ are different and depend on $d$. The increase of $L_{\text {opt }}$ with the increase of $d$ is observed. These values seem to be related to the peaks of compressibility and permeability as functions of $L$, which maximizes the dissipation of acoustic waves.

Different optimal parameters are found for the soft elastic frame made of silicone rubber. In this case, the optimal porosity does not tend to be maximum but instead takes low values whereas the cell size appears to be high. The optimal values $J_{\text {opt }}$ does not increase much but the behavior of absorption coefficient changes quantitatively as seen in Fig. 4. We observe the same peaks as in the case of epoxy frame but these are shifted to lower frequency band and occur more often. This phenomenon is caused by the resonance of the frame which becomes more pronounced for soft skeletons.

\section{Conclusion}

A framework to determine the acoustic absorption of a poroelastic panel from microstructural parameters has been presented. The cylindrical pores are saturated with heat conducting air which enables essential attenuation of acoustic energy. The effective properties are derived via a homogenization procedure for different types of matrix materials: epoxy (stiff) and silicone rubber (soft). The homogenisation technique is rigorously defined and the simple pore geometry model considered in this study can be validated by the self-consistent approach or by estimating upper and lower bounds of parameters [6]. For more complex microstructure porous media, the validation of homogenized models could be done by comparing with results obtained from micro FE simulation on the model with its real microstructural geometry [7].

The absorption coefficients of poroelastic layers are computed by using effective properties for rigid and elastic frames of considered materials. The comparison of absorption coefficient for these cases shows that a poroelastic material with stiff skeleton has similar acoustic performances to the one modeled with a perfectly rigid a)
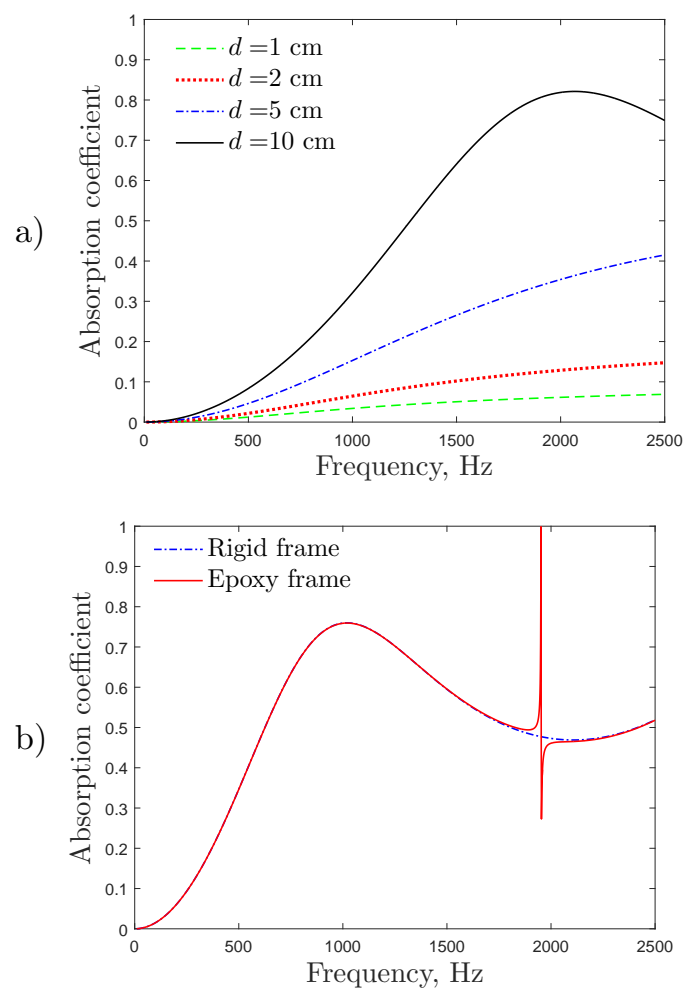

Figure 3: Absorption coefficients obtained with optimal design parameters: (a) Cases $d=1,2,5,10 \mathrm{~cm}$ : the difference between values obtained for rigid and epoxy frames is negligible; (b) Case $d=20 \mathrm{~cm}$ : the difference between values obtained for rigid and epoxy frames is found at $f \approx 2000 \mathrm{~Hz}$

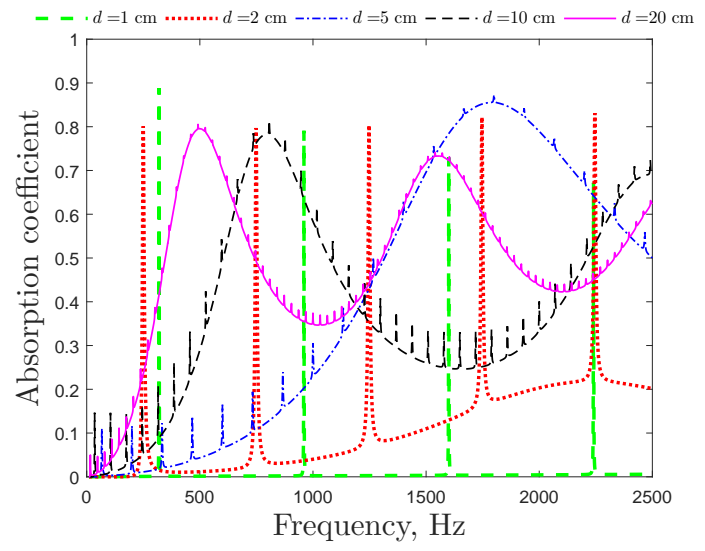

Figure 4: Absorption coefficients obtained with optimal design parameters of silicone rubber frame for $d=1,2,5,10,20 \mathrm{~cm}$.

frame hypothesis. For the material with the epoxy frame, a fast change of the absorption coefficient is observed around a certain frequency for the particular layer thickness. Outside this range, the differences are not significant and consequently, same optimal cell size and porosity are obtained. The soft matrix material (silicone rubber) shows to have distinct results for the acoustic absorption with multiple peaks within the studied range of frequencies. The optimal cell size appears to be higher than in 
the other considered cases, however, the optimal porosity is lower. The obtained results on optimisation are consistent with the observations of other researches, see e.g [17], reporting the improvement of absorption for circular inclusions and their arrangement in rigid porous materials.

In this study, only homogeneous porous plate with cylindrical pores has been studied for illustration and validation purposes. Due to the simplicity of the chosen pore geometry, the absorption properties could only be improved at relatively large layer thickness. Using foam materials instead of elastic skeletons or taking the resonant inclusions can significantly improve the performance of an acoustic absorber of even small thickness as the studies show, for instance, in $[17,19]$. Therefore, further studies to consider different cell geometries and/or heterogeneous plates, e.g. plates made of multi-layered, functionally-graded or periodic mixture of poroelastic materials with contrasts $[7,30]$, can be performed using the developed optimization procedure. Moreover, the presented optimization algorithm can be extended to consider the combination between various physical material properties with microstructural characteristics, e.g. higher rigidity or additional heat insulation. Acknowledgments This work has benefited from a French government grant managed by ANR within the frame of the national program of Investments for the Future ANR-11-LABX-022-01 (LabEx MMCD project).

\section{References}

[1] J. Allard, N. Atalla, Propagation of sound in porous media: modelling sound absorbing materials, John Wiley \& Sons, 2009.

[2] M. Kaviany, Principles of heat transfer in porous media, Springer Science \& Business Media, 2012.

[3] M. A. Biot, Theory of elasticity and consolidation for a porous anisotropic solid, Journal of Applied Physics 26 (2) (1955) 182185.

[4] E. Detournay, A. H.-D. Cheng, Fundamentals of poroelasticity, in: Analysis and Design Methods, Elsevier, 1995, pp. 113-171.

[5] S. R. Pride, A. F. Gangi, F. D. Morgan, Deriving the equations of motion for porous isotropic media, The Journal of the Acoustical Society of America 92 (6) (1992) 3278-3290.

[6] J.-L. Auriault, C. Boutin, C. Geindreau, Homogenization of coupled phenomena in heterogenous media, Vol. 149, John Wiley \& Sons, 2010.

[7] V.-H. Nguyen, E. Rohan, S. Naili, Multiscale simulation of acoustic waves in homogenized heterogeneous porous media with low and high permeability contrasts, International Journal of Engineering Science 101 (2016) 92 - 109.

[8] E. Rohan, V.-H. Nguyen, S. Naili, Wave propagation in a strongly heterogeneous elastic porous medium: Homogenization of biot medium with double porosities, Comptes Rendus Mecanique 344 (2016) 569-581.

[9] M. Hori, S. Nemat-Nasser, On two micromechanics theories for determining micro-macro relations in heterogeneous solids, Mechanics of Materials 31 (10) (1999) 667-682.

[10] C. K. Lee, C. C. Mei, Thermal consolidation in porous media by homogenization theory. II. Calculation of effective coefficients, Advances in Water Resources 20 (2-3) (1997) 145-156.

[11] C. van Duijn, A. Mikelic, M. Wheeler, T. Wick, Thermoporoelasticity via homogenization. I. Modeling and formal two-scale expansions, International Journal of Engineering Science 138 (2019) 1-25.
[12] T. Yamamoto, S. Maruyama, K. Terada, K. Izui, S. Nishiwaki, A generalized macroscopic model for sound-absorbing poroelastic media using the homogenization method, Computer Methods in Applied Mechanics and Engineering 200 (1-4) (2011) 251264.

[13] X. Olny, C. Boutin, Acoustic wave propagation in double porosity media, The Journal of the Acoustical Society of America 114 (1) (2003) 73-89.

[14] J. S. Lee, E. I. Kim, Y. Y. Kim, J. S. Kim, Y. J. Kang, Optimal poroelastic layer sequencing for sound transmission loss maximization by topology optimization method, The Journal of the Acoustical Society of America 122 (4) (2007) 2097-2106.

[15] E. Lind-Nordgren, P. Göransson, Optimising open porous foam for acoustical and vibrational performance, Journal of Sound and Vibration 329 (7) (2010) 753-767.

[16] C. Perrot, F. Chevillotte, R. Panneton, Bottom-up approach for microstructure optimization of sound absorbing materials, The Journal of the Acoustical Society of America 124 (2) (2008) 940-948.

[17] J.-P. Groby, O. Dazel, A. Duclos, L. Boeckx, L. Kelders, Enhancing the absorption coefficient of a backed rigid frame porous layer by embedding circular periodic inclusions, The Journal of the Acoustical Society of America 130 (6) (2011) 3771-3780.

[18] T. Weisser, J.-P. Groby, O. Dazel, F. Gaultier, E. Deckers, S. Futatsugi, L. Monteiro, Acoustic behavior of a rigidly backed poroelastic layer with periodic resonant inclusions by a multiple scattering approach, The Journal of the Acoustical Society of America 139 (2) (2016) 617-629.

[19] C. Lagarrigue, J.-P. Groby, O. Dazel, V. Tournat, Design of metaporous supercells by genetic algorithm for absorption optimization on a wide frequency band, Applied Acoustics 102 (2016) 49-54.

[20] C. Lagarrigue, J. Groby, V. Tournat, O. Dazel, O. Umnova, Absorption of sound by porous layers with embedded periodic arrays of resonant inclusions, The Journal of the Acoustical Society of America 134 (6) (2013) 4670-4680.

[21] M. R. Stinson, The propagation of plane sound waves in narrow and wide circular tubes, and generalization to uniform tubes of arbitrary cross-sectional shape, The Journal of the Acoustical Society of America 89 (2) (1991) 550-558.

[22] T. Levy, E. Sanchez-Palencia, Equations and interface conditions for acoustic phenomena in porous media, Journal of Mathematical Analysis and Applications 61 (3) (1977) 813-834.

[23] J.-L. Auriault, L. Borne, R. Chambon, Dynamics of porous saturated media, checking of the generalized law of Darcy, The Journal of the Acoustical Society of America 77 (5) (1985) 16411650 .

[24] C.-N. Wang, J.-H. Torng, Experimental study of the absorption characteristics of some porous fibrous materials, Applied Acoustics 62 (4) (2001) 447-459.

[25] B. G. Compton, J. A. Lewis, 3D-printing of lightweight cellular composites, Advanced Materials 26 (34) (2014) 5930-5935.

[26] M. Hirsekorn, P. Delsanto, N. Batra, P. Matic, Modelling and simulation of acoustic wave propagation in locally resonant sonic materials, Ultrasonics 42 (1-9) (2004) 231-235.

[27] M. Zainulabidin, M. Rani, N. Nezere, A. Tobi, Optimum sound absorption by materials fraction combination, International Journal of Mechanical \& Mechatronics Engineering 14 (2) (2014) 118-121.

[28] R. Hooke, T. A. Jeeves, "Direct search"solution of numerical and statistical problems, Journal of the Association for Computing Machinery (JACM) 8 (2) (1961) 212-229.

[29] L. Wen, L. Gao, X. Li, L. Zhang, Free pattern search for global optimization, Applied Soft Computing 13 (9) (2013) 3853-3863.

[30] E. Rohan, V.-H. Nguyen, S. Naili, Numerical modelling of waves in double-porosity Biot medium, Computers \& Structures (in press). 